\title{
Desarrollando competencias en el modelo escuela nueva. Programa Universidad en el campo, UNAN-Managua, Nicaragua
}

Julio César Laguna Gámez ${ }^{1}$

\section{RESUMEN}

E1 Modelo Escuela Nueva, del programa Universidad en el Campo, define desarrollar competencias en discentes en formación, para la implementación de los pilares de la educación definidos por la UNESCO, que son aprender a conocer, aprender a ser, aprender a hacer, aprender a convivir y aprender a convivir con el medio ambiente. En este trabajo se realizaron entrevistas a docentes que imparten docencia al grupo de Desarrollo Rural Sostenible en el Municipio Tuma La Dalia y a un grupo focal de 13 estudiantes. Los principales resultados en los docentes, en el aprender a conocer, tienen buena formación técnica, implementan metodologías activas pero todavía en varios momentos aplican educación tradicional, presentan debilidades en la evaluación del conocimiento. La investigación se aplica pero con limitantes, no se hace la aplicación teórica a la solución de la problemática identificada. El aprender a ser, tiene que ver con comportamiento y valores que empiezan con lo que trasmite el facilitador. El aprender a convivir, tiene que ver con la tolerancia, situación que se cultiva de forma sistemática. En el aspecto de aprender a convivir con el medio ambiente, se ha hecho mucho énfasis, apropiando al estudiantado en una sólida conciencia ambientalista.

Palabras claves: Aprender a conocer, aprender a ser, aprender a hacer, aprender a convivir, aprender a convivir con el medio ambiente.

Recibido: 12 de mayo de 2016

Aceptado: 25 de agosto de 2016

1 Docente Titular UNAN FAREM Matagalpa; estudiante de Doctorado en Desarrollo Rural. Correo Electrónico: jlagunagamez@yahoo.es 


\title{
Developing competencies in the New School Model. Rural University program, UNAN-Managua, Nicaragua
}

\begin{abstract}
The New School Model of the Rural University (Universidad del Campo) aims to develop competencies in students, for the implementation of the education basis defined by UNESCO; namely learn to know, learn to be, learn to make, learn to live and learn to live in harmony with the environment. This work featured interviews to teachers from the Sustainable Development Program in the Municipality of Tuma, La Dalia, and a focus group with 13 students. The main results reveal that the teachers hold a good technical formation, they implement active methodologies inside the classroom; however, they still apply a traditional methodology in some moments. Moreover, they are weak regarding evaluation procedures. They conduct research in a limited extent, and they do not apply theory in relation to problems identified. Learning to be deals with a behavior and values that start with what is conveyed by the facilitator. Learning to be deals with tolerance, which is systematically developed. When it comes to learning to live in harmony with the environment, an environmental conscience has been highlighted.
\end{abstract}

Key words: Learn to know, learn to be, learn to make, learn to live, learn to live in harmony with the environment. 


\section{INTRODUCCIÓN}

La Universidad Nacional Autónoma de Nicaragua, Managua impulsa el programa Universidad en el Campo, que es un proyecto innovador de formación técnica profesional en desarrollo rural sostenible. Este programa fue diseñado para los jóvenes del sector rural de escasos recursos económicos, como una opción para alcanzar la educación superior, lo que lo hace un programa innovador y atractivo, que contribuye a disminuir la deserción y la emigración de los jóvenes estudiantes hacia los centros poblados (Universidad de Caldas, 2011).

La carrera impulsada es Desarrollo Rural Sostenible, se basa en desarrollar competencias integrales enfocadas desde lo cognoscitivo, lo procedimental y lo actitudinal. Promueve un proceso de aprendizaje cooperativo y personalizado centrado en el estudiante, en la formación de valores, en un nuevo rol del docente como orientador y facilitador y en un nuevo concepto de guías de aprendizaje, versus la educación tradicional, pasiva y autoritaria (Colbert, 1999).

Los ejes curriculares de la carrera en Desarrollo Rural Sostenible, están orientados a generar competencias integrales que le permita a los estudiantes aprender a conocer, aprender a hacer, aprender a vivir juntos, aprender a vivir juntos sin comprometer el ambiente. Los ejes curriculares pretende desarrollar las competencias para la formación integral del desarrollo rural, la producción agropecuaria, que tiene como ejes transversales la búsqueda de modelos sustentables desde la perspectiva social, económica y ecológica (Universidad de Caldas, 2011).

Es necesario eliminar de los currículos los contenidos excesivamente teóricos, abstractos y con baja probabilidad de ser utilizados en la vida, en el trabajo rural. En su lugar, deberían ser incluidos contenidos más prácticos y aplicables en la solución de los problemas del territorio rural (Lacki, SF).
Las competencias que se desarrollan en la formación de los estudiantes se orientan a la calidad y nivel de desempeño que incluye habilidades, conocimientos, destrezas y actitudes que se requieren para planificar, ejecutar, controlar y desarrollar de la mejor forma una tarea. Los docentes facilitadores para que desarrollen competencia en sus discentes, deben de apropiarse de competencias para desarrollar su trabajo que debe iniciar con su dominio en el área de conocimiento científico, pertenencia e identificación con el campo educativo y sus diversas estrategias de transmisión del conocimiento, interés en su desarrollo sistemático, incentivar el espíritu innovador, la calidad y la pertinencia social, orientado a que su trabajo impacte en la formación de los estudiantes, en el dominio del saber hacer en las diferentes prácticas objetos de dominio, que los haga competentes y competitivos (DGESPE, 2009).

En el modelo Escuela Nueva se reconoce que el aprender hacer es indispensable en su aplicación, que es un proceso continuo en el cual se pueden identificar varios momentos. El primero está relacionado con reconocer que los estudiantes tienen un saber, no llegan a la institución educativa carentes de conocimientos, habilidades o valores, ya sea porque han desarrollado competencias en la interacción en el hogar, con los padres, en su contacto con la unidad productiva, en las acciones pedagógicas realizadas en años anteriores, o en los múltiples saberes que provienen de las nuevas tecnologías de la información y la comunicación (Ministerio de Educación Nacional, 2010).

A diferencia de la educación tradicional o bancaria, donde el trabajo que muchas veces se fomenta es exclusivamente el desarrollo individual, que trae como consecuencia la promoción de actitudes egoístas, se presenta la propuesta de trabajo colaborativo que busca la unión de las competencias de cada estudiante para el logro de objetivos de aprendizaje. Para el modelo educativo Escuela Nueva el trabajo colaborativo es una de las estrategias fundamentales, donde los estudiantes 
tienen la posibilidad de interactuar con distintas perspectivas frente a un problema (Johnson, 1999).

Un componente indispensable en la creación de un ambiente educativo para la formación de la autonomía consiste en realizar acciones comunicativas favorables para que los estudiantes comprendan que el aprendizaje no es lo que acontece exclusivamente en el salón de clase (Duarte, 2010), sino que fuera de la Universidad se encuentran diversos escenarios para el desarrollo de sus competencias. Proponer actividades que trasciendan el aula de clase contribuye a que los estudiantes le encuentren sentido a los conocimientos que allí se trabajan. Las actividades que vinculan la vida escolar y la vida fuera de la institución educativa crean un clima que motiva a los estudiantes a aprender, pues entienden que el conocimiento y el desarrollo de habilidades y valores les sirven para orientarse y desempeñarse en la cotidianeidad.

El modelo Escuela Nueva se basa en el diseño de cartillas de aprendizaje que poseen una secuencia metodológica que parte de la vivencia, se refuerza con la fundamentación científica, luego se hace la ejercitación, para pasar a aplicación práctica y se refuerza con complementación según las inquietudes que se hayan despertado (Arenas, 2016). En este sentido, las actividades de vivencia, ejercitación y aplicación de cada unidad motivan a los estudiantes para el saber hacer, para que desde la transferencia de competencias, mejoren la calidad de vida de su familia a través del desarrollo de todo tipo de proyectos. La fundamentación científica va orientada al saber conocer. Y todos los momentos metodológicos levan transversal el saber convivir, el saber ser y el respeto al medio ambiente. El estudiante realiza su proceso de formación desde la comunidad y para la comunidad, desde su experiencia con su familia y para obtener una práctica transformada con ellos.

Según la Universidad de Caldas (2011), estos momentos se describen de la siguiente manera:

\section{A. Vivencias:}

Parte de una etapa de exploración en la que se identifican los conocimientos previos, las actitudes y expectativas, sea cual fuese su grado de procedencia y nivel de formación. Esta experiencia de valoración de aprendizaje acumulado o inventario de logros de vida personal, lo denominamos vivencias. Nivel de desempeño: reconocimiento (dimensión cognitiva). Generalmente desarrollado en su seno familiar y en su experiencia comunitaria.

\section{B. Fundamentación científica:}

Logrado el primer momento de reconocimiento de aprendizajes adquiridos (vivencias), el proceso continua hacia el acercamiento a nivel de la conceptualización a través de una etapa de fundamentación y documentación científica, bien sea por medio de lecturas, documentos de apoyo $u$ otros, en la cual se ofrece al estudiante información teórica sobre principios, leyes, normas, conceptos que explican, dan razón o fundamentan el tema central, mediante formas didácticas y pedagógicas propias de su edad y del grado de abstracción ideológica.

La fase de fundamentación científica, amplía, explica, clasifica, da respuestas científicas a los conocimientos acumulados; retoma y profundiza las vivencias del estudiante. En síntesis, la lectura o la información documentada, aporta al estudiante nuevos conocimientos, científicamente válidos para que al asimilarlos los reutilice, compruebe y aplique.

Como ayuda para la asimilación de los conceptos abordados en la fase de fundamentación científica, el módulo o guía modular puede incluir un glosario de términos técnicos y científicos (no superior a 30 términos claves, relacionados en la fundamentación científica). Nivel de desempeño: reconocimiento (dimensión cognitiva).

\section{Ejercitación}

La actividad de ejercitación permite la utilización de los conocimientos adquiridos en las fases anteriores, 
se habilita al estudiante para el manejo de operaciones formales que transcienden notablemente los procesos mentales de asimilación, concreción, adaptación y aplicación de lo aprendido.

El análisis, la reflexión, la síntesis, la argumentación, la conceptualización son actividades propias de la ejercitación, ya que se busca a través de ellas que el estudiante desarrolle habilidades y destrezas para la identificación y concreción de situaciones problemáticas, abarque contextos, perciba causas, efectos y hechos.

Se hace énfasis en la reconstrucción de situaciones, en la organización de la información escrita y en el buen manejo del análisis y de la síntesis, así como en la descripción de eventos y en la representación de sucesos cotidianos que conforman la vida social, cultural y familiar del estudiante. En este ejercicio de orden práctico, juega un papel definitivo la capacidad creativa del estudiante, su habilidad para el manejo de la lectura comprensiva y la investigación.

La actividad de ejercitación pretende además, despertar en el estudiante cualidades que le garanticen un buen nivel de socialización y de integración, una actitud solidaria que le desarrolle habilidades de liderazgo, de altruismo y capacidad de tolerancia y de respeto por las ideas e iniciativas de sus compañeros (dimensión actitudinal).

La ejercitación del conocimiento debe conducir al estudiante al hallazgo de una posición de equilibrio que armonice dentro de una relación de desajuste - ajuste para la asimilación por motivación e incentivación, de nuevas experiencias de aprendizaje. Igualmente se rescata en esta fase, la técnica de la pedagogía del error (ensayo - error) al permitir que el estudiante confronte, construya y reconstruya alternativas para abordar situaciones nuevas.
De igual manera surge como otra de las posibilidades creativas de esta actividad, la inducción del estudiante en forma progresiva y consciente en la tarea investigativa, haciendo uso de estrategias que le garanticen su participación dinámica en los procesos de interaprendizaje y en la conformación de su propio estilo, en el manejo de su propio ritmo y metodología de trabajo. Nivel de desempeño: uso del conocimiento (dimensión procedimental)

\section{Aplicación}

En este momento se fortalece la actividad investigativa, puesto que lleva al estudiante a comprometerse e incidir sobre situaciones problemáticas vividas dentro y fuera del aula y que transcienden a su realidad social. Estas acciones son ejecutadas por el estudiante a través de proyectos que siguen los pasos de la investigación científica. El estudiante diseña individualmente o en la mesa de trabajo, estrategias que le permiten caracterizar y definir el "problema", los procedimientos y las técnicas de manejo, con el fin de lograr, en la práctica, su tratamiento adecuado. Este proceso de búsqueda facilita la construcción de alternativas de solución que se van reforzando progresivamente con el alcance de los objetivos previstos en cada unidad. Se involucra a la comunidad y a la familia en particular en el proceso de formación de competencias.

La extrapolación como método de trabajo facilita al estudiante el desarrollo de habilidades para el dominio de transferencia de conocimientos, y para la asimilación de vivencias y experiencias análogas. Este proceso secuencialmente realizado garantiza la reafirmación de los saberes en el estudiante y la dinamización de acciones comunitarias en procura de transformaciones sociales mucho más consolidadas.

Los proyectos procuran además mediante objetivos bien formulados, aplicar tecnología para la capacitación del estudiante, la escuela y la comunidad en el hacer y en el producir, dentro de una dinámica científica, rigurosa y eficaz, en términos de su utilización inmediata para encontrar respuestas a problemas del medio. 
Dichos proyectos estarán orientados a la producción en áreas agropecuarias, de desarrollo rural, académicas o comunitarias según su enfoque y de acuerdo con las necesidades detectadas por el alumno y por la institución. Este tipo de actividades en la zona de influencia del medio, incorpora la adecuación y uso de tecnologías por parte de las instituciones que rodean el centro educativo. Nivel de desempeño: Proponer (dimensión procedimental).

\section{E: Complementación o Ampliación}

En esta parte del proceso el estudiante ha desarrollado habilidades y destrezas mentales, que dentro de la relación ajuste - desajuste, generan una nueva situación de desequilibrio, la cual a su vez crea nuevas necesidades de aprendizaje. Corresponde entonces al módulo suministrar y ampliar las referencias bibliográficas y conceptuales que permiten restablecer el estado de equilibrio y reafirmar nuevos aprendizajes. La actividad de complementación induce al estudiante a nuevas exploraciones y confrontaciones para la construcción y aplicación de nuevos conocimientos, este propósito se logra con la actividad de complementación, al inducir al estudiante en nuevas exploraciones. Nivel de desempeño: investigar (dimensión cognitiva). Nota: la dimensión actitudinal, se fundamenta, valora y realimenta de manera transversal durante todo el proceso metodológico.

De ahí que se propone realizar evaluaciones para obtener información sobre los aprendizajes que tienen los estudiantes antes de enfrentar un nuevo problema o elaborar un concepto. Implementar este tipo de evaluación favorece la motivación y la curiosidad de los estudiantes para que asuman con entusiasmo el nuevo desafío educativo y adecuen la estrategia de enseñanza de acuerdo con las necesidades del contexto. Es importante crear un ambiente favorable para que los estudiantes y el docente puedan explicitar sus expectativas frente al nuevo reto educativo.

En el avance del proceso de enseñanza aprendizaje y en la utilización de las guías modulares y su estructura metodológica, es importante que los estudiantes se organicen en equipos de trabajo, pero donde desarrollen un papel activo en la construcción colectiva de conocimientos, definiendo una serie de conjuntos de roles orientados a fortalecer la actividad grupal en un ejercicio de liderazgo compartido.

Según Mogollón y Solano (2011) los roles que deben tener los estudiantes y que deben de rotar sistemáticamente en el desarrollo del modelo Escuela Nueva son:

- Coordinador: su tarea es organizar al grupo, incentivar, motivar, asume el liderazgo para que el trabajo se realice con responsabilidad y con la debida entrega, para que se desarrolle de la mejor manera. Evita las distracciones y enrumba al grupo al cumplimiento de las metas trazadas, solicita al facilitador cuando se enfrenta dudas o problemas.

- El administrador del tiempo: es quien controla el tiempo, durante el desarrollo de la actividad va indicando el tiempo restante, para ajustarse al avance que se lleva. Participa activamente en el desarrollo del trabajo. Su trabajo es de suma importancia para no extenderse más del tiempo indicado por las guías modulares o el facilitador.

- El relator: debe demostrar la habilidad y competenciaparala redacción, así como capacidades para recoger conceptos, inquietudes, mediación entre los compañeros del grupo y el facilitador y desarrolla capacidad de construir textos. El relator desarrolla habilidades comunicativas, tanto orales como escritas.

- Investigador: en el desarrollo de este roll, el estudiante es acucioso y dinámico, para proponer donde buscar la información requerida, pude ser en la guía modular, en literatura disponible, en Internet, consultando a personas, instituciones, organismos, personalidades, etc.

- El ayudante: es el estudiante que busca y garantiza los materiales, se encarga de administrar su utilización y el cuidado de marcadores, 
papelógrafos, cintas adhesivas, uso de guías, de texto, de material vegetativo, etc.

Cada uno de los diferentes rolles desarrollado en los equipos de trabajo deben de ser rotado en la siguiente actividad grupal, para que todos desarrollen las competencias que permiten las actividades grupales.

Pero metodológicamente, el modelo no solo trabaja con los equipos de trabajo, sino que para propiciar el desarrollo de competencias, se debe pensar en la formación desde el saber-hacer, en forma efectiva y en los diferentes ámbitos del quehacer profesional y social. Ello requiere conocer las necesidades, a efectos de relacionarlas con los métodos de aprendizaje. Esto supone modificaciones en el rol del docente facilitador, quien debe reconocer, reflexionar y revalorizar las situaciones de aprendizaje, desde la planificación hasta la evaluación en función del desarrollo de las competencias que se incluyan en el currículo (Conde, 2008).

Las competencias se refiere a las atribuciones, incumbencia de tareas o funciones, donde una cosa es "ser capaz de" (poseer una habilidad, destreza, potencialidad) y otra "ser competente para" (capacidad para utilizar); la diferencia es un proceso de formación (Ramón, 2010).

La competencia es el conjunto de conocimientos, actitudes, disposiciones y habilidades (cognitivas, socioafectivas y comunicativas) relacionadas entre sí para facilitar el desempeño flexible del estudiante en la realización de diversas actividades prácticas (MEN/ ASCOFADE, 2005).

Competencia es utilizar lo que se sabe, la teoría aprendida, es poner el saber en acción. La práctica educativa debería insistir en replantear la adquisición de conocimientos que no tienen utilidad para el desarrollo de proyectos de vida, pues se trata de poner en acción recursos como el saber, el saber hacer, las actitudes, procedimientos, habilidades, comportamientos sociales y personales, que se combinan para solucionar problemas (Ramón, 2010).

Un profesional en desarrollo rural sostenible debe de desarrollar una competencia, frente al advenimiento de los transgénicos, poder analizar que adquirir la nueva semilla y sembrarla, construye la muerte de la diversidad de especies de ese cultivo, frente al que sus antepasados adaptaron, cuidaron y cosecharon durante mucho tiempo. Saber que esa semilla transgénica promete resistencia a plagas y mejores rendimientos productivos, pero ser capaz de preguntarse ¿a cambio de qué? Es decir, su análisis para llegar a decidir, aun lo complejo que sea, lo que demanda mayor información para poder decidir con autonomía y certeza (Mejía, 2006) pero solo cuando se hace se ha desarrollado una competencia.

Las competencias se pueden dividir en dos tipos: competencias genéricas, que en principio son independientes del área de estudio y competencias específicas para cada área temática. Las competencias se obtienen normalmente durante diferentes unidades de estudio y por tanto pueden no estar ligadas a una sola unidad (Bravo, 2007).

Pero los docentes debemos prepararnos para ser facilitadores y formadores de competencias, y evolucionar como afirman Hidalgo y Marín (2003) que describen los paradigmas de la educación. Iniciando con el Paradigma Positivista donde el rol del docente es como evaluador para verificar el dominio de los contenidos. En el Paradigma Postpositivista su rol pasó a ser descriptor de información, selector de contenidos educacionales pero se mantuvo el énfasis en la creación de instrumentos para medir conocimientos. En el paradigma crítico, tenemos un docente más humanista que hace énfasis en metodologías que favorecen el diálogo, la mediación y la transferencia de realidades, mientras que su rol es de proveedor de información y emisor de juicios valorativos para llevar a cabo procesos de autoevaluación. En el Paradigma Emergente el docente pasa a ser promovedor de 
construcción de realidades, tomando en cuenta la individualidad de cada ser, la subjetividad de cada proceso y la participación del estudiante como centro del proceso educativo.

En el paradigma emergente el docente se destaca como actor principal de este escenario educativo, quien debe cambiar su concepción del proceso enseñanza-aprendizaje, dejando de ser el profesor de clases magistrales para convertirse en facilitador del conocimiento, orientadory guía de los alumnos, pasando además a ser un experto en manejo de herramientas de comunicación, promotor de la interacción profesoralumno, alumno-alumno, y alumno-contenidos, y capaz de cambiar los materiales educativos por materiales que promuevan la interacción con estos contenidos (Hidalgo y Marín 2003).

El sistema educativo, ahora, está centrado en una formación fundamentada en competencias, donde el centro de todo proceso lo constituye el conocer y desarrollar habilidades partiendo de tareas definidas, el docente debe desempeñar un papel que va mas allá de un dador de información, y para ello debe convertirse en un ser integral capaz de implementar los criterios que definen un buen desempeño o desempeño idóneo: el saber ser, el saber conocer, el saber hacer y el saber convivir (Pérez, 2011).

El saber conocer es un conjunto de conocimientos sistematizado, lógicamente ordenados en libros, revistas, archivos o manuscritos; referidos todos ellos a un campo específico del conocimiento, los saberes se refieren a hechos, situaciones y objetos; los hechos pueden ser físicos o naturales e incluso sociales; las situaciones se desarrollan en los contextos y en ellas participan sujetos sociales; en este sentido, los sujetos se convierten en objetos de conocimiento.

Por su parte, saber hacer es resolver problemas, los problemas de orden práctico demandan soluciones prácticas; saber hacer es la demostración de congruencia entre lo que se dice que se sabe y lo que se hace. El saber ser, es el cultivo de los valores, principios, responsabilidades, virtudes, cualidades, capacidades, aptitudes, convicciones, ideas, ideales, creencias, opiniones, comportamiento, etc. (Mialaret, 1977), el enfoque de competencias implica cambios y transformaciones profundas en los diferentes niveles educativos, y seguir este enfoque es comprometerse con una docencia de calidad, buscando asegurar el aprendizaje de los estudiantes, se pasa de la formación en conocimientos memorísticos a la formación de desempeños ante situaciones y problemas con sentido para las personas en el saber hacer, saber convivir, saber conocer y saber ser para afrontar los retos y tareas del contexto mediante la interrelación de facilitador, estudiantes y entorno.

\section{MATERIALES Y MÉTODOS}

El trabajo se realizó con docentes facilitadores y estudiantes de la carrera de Desarrollo Rural Sostenible, del Programa UNICAM del municipio del Tuma La Dalia, impulsado por la FAREM Matagalpa, de la UNAN Managua durante el segundo semestre del 2015.

La investigación es no experimental, de corte transversal o transeccional y del tipo descriptivo con enfoque cuali-cuantitativo, lo que permite describir las competencias de los docentes para la implementación del modelo Escuela Nueva en la carrera de Desarrollo Rural Sostenible.

El universo del estudio son los 56 estudiantes de la primera cohorte de la carrera de Desarrollo Rural Sostenible, formando la muestra con la integración de forma voluntaria de 13 estudiantes para la realización de un grupo focal participativo. Se utilizaron preguntas guiadoras para la realización del grupo focal. Se grabó las participaciones del grupo focal, para después proceder con la redacción de los resultados.

Se entrevistaron a seis docentes facilitadores que imparten clase, seleccionados al azar y una vez que 
se le propuso los objetivos del estudio, se respetó el principio de voluntariedad para ser incluidos. Para la realización de las entrevista se diseño una guía de preguntas abiertas. Para la realización de la entrevista se realizaron grabaciones para después redactar los resultados. Los resultados se describen a partir de las opiniones según la percepción de los estudiantes y las entrevistas brindadas por los docentes.

Las variables que se estudiaron fueron las capacidades de los docentes en los aspectos del saber conocer, saber hacer, saber ser, saber convivir y saber convivir con el medio ambiente.

\section{RESULTADOS Y DISCUSIÓN}

Los docentes facilitadores son el pilar fundamental en el impulso del modelo Escuela Nueva, pero demanda un conjunto de cualidades, capacidades y competencias para desarrollar de manera eficiente su práctica pedagógica. Los principios desarrollados en el proceso educativos son:

\section{Saber conocer}

Saber conocer es que los docentes facilitadores deben tener una preparación profesional solida para llevar a cabo exitosamente su labor; es decir tener pleno conocimiento teórico práctico de las asignaturas que imparte y un sólido conocimiento de los contenidos a intercambiar.

Los estudiantes afirman que todos los docentes facilitadores que imparten docencia en la carrera de Ingeniería en Desarrollo Rural Sostenible, son profesionales con altas capacidades de conocimiento teórico práctico. Los contenidos facilitados por los docentes son bien valorados. Los estudiantes expresan que los docentes dominan el área de conocimiento de la asignatura que imparten.

El saber conocer tradicionalmente se ha conocido como el hecho de trasmitir conocimiento, pero también abarca el vínculo de la trasmisión de los conocimientos teóricos desde un enfoque investigativo, conduciendo a una reflexión desde el aula de estudio con la investigación, que incorpore al proyecto educativo, no exclusivamente como aprendizaje de metodologías y técnicas de aplicación, sino hacia el análisis y solución de problemas del contexto social, con una educación problematizadora. La aplicación de la investigación se basa en encontrar el problema, realizar observaciones del medio de forma crítica, en la formulación de hipótesis. Hasta este punto los estudiantes y los mismos docentes afirman que se realizan en el aula de clase y en los diferentes momentos metodológicos que se desarrollan.

Pero los estudiantes indican que se hace evidente la falta de interrelación con la discusión teórica del problema y con mayor dificultad con la demostración científica del mismo. Aunque los docentes afirman realizarlo, si expresan que para la aplicación de estos aspectos requieren de una mayor preparación y estudios que fortalezcan sus conocimientos teóricos y para mantenerse actualizados. Los profesores afirman están de acuerdo que para poder acrecentar y actualizar constantemente los conocimientos necesitan poseer un contante vinculo con las investigaciones que se dan en el propio campo del saber al que pertenecen. La investigación incluye los aportes actualizados en la propia especialidad, a nivel nacional e internacional, $\mathrm{y}$ en las relaciones interdisciplinares, constituyendo una de las fortalezas que debe cuidar la docencia universitaria.

Otro aspecto importante es el fomento de las innovaciones productivas tecnológicas, los docentes y estudiantes afirman que son fáciles de aplicar en las asignaturas profesionalizantes productivas $\mathrm{o}$ en aquellas que terminan con trabajos de cursos. Pero hay asignaturas que se facilitan para impulsar innovaciones productivas tecnológicas, pero que no se optimizan. Las tecnologías alternativas son vitales para el desarrollo del territorio rural, pero en algunas ocasiones se trasmite tecnologías alternativas que no 
tienen aplicabilidad a las condiciones socioambientales del territorio donde se esta infiriendo. Los estudiantes piden que se debe revisar las tecnologías que se quieren transferir debido a que algunas son de aplicación para trópico seco y los grupos que se están atendiendo son de trópico semihúmedo a trópico húmedo, o bien son tecnologías aplicables a otras realidades ajenas a las propias.

Sobre las formas evaluativas del conocimiento, aplicadas por los docentes se puede considerar como innovadoras, pero que todavía conserva de las formas tradicionales de evaluación. La mayoría de los docentes facilitadores tienen claridad sobre los objetivos de la evaluación, la que es utilizada como diagnóstico para detectar debilidades y establecer acciones de superación en los estudiantes, a partir de los conocimientos facilitados. Pero existen docentes que considera que las evaluaciones es una metodología que permite obtener una calificación para el estudiante o como la forma de ponderar el grado de asimilación de los conocimientos teóricos. Los estudiantes recomiendan que las evaluaciones deben de hacer hacia la parte practica, pero hay docente que evalúan esperando que los examinados repitan textualmente lo trasferidos por los docentes.

Las formas evaluativas son muy diversas que van desde participación en clase, seminarios, debates, trabajos en grupos, exposiciones, trabajos escritos como cuestionarios, análisis de lecturas, investigaciones documentales, investigaciones en las comunidades, con instituciones, exposiciones sobre experiencias comunitarias, trabajos individuales $\mathrm{y} / \mathrm{o}$ grupales orientados por las guías modulares, investigaciones e innovaciones productivas tecnológicas, trabajos prácticos en campo, desarrollo de competencias genéricas o especificas, pruebas cortas, examenes parciales, trabajos de cursos, propuestas de proyectos, etc. Entre estas estrategias evaluativas se encuentra que hay elementos comunes como: acciones con propósito definido, estrategia para reforzar el conocimiento y hacer seguimiento. Los estudiantes indican que no se hace una atención individualizada, a partir de las debilidades que algunos poseen. Según los estudiantes en determinado momentos algunos docentes han afirmado, que ellos no son los culpables de las debilidades que poseen los estudiantes y que son conocimientos que deben de haber sido desarrollados anteriormente. Aquí debe de hacerse un compromiso para mejorar estos vicios de la educación tradicional.

Sobre el nivel de comprensión y conocimiento del modelo educativo Escuela Nueva, la mayoría de los docentes tienen niveles diferentes de dominio del modelo, generalmente se apropian de la metodología al leer y seguir los pasos metodológicos que se encuentran en las estructuras de las guías modulares, que inician con la vivencia, continúan con la fundamentación científica, posterior con la ejercitación y la aplicación y concluyen con la complementación.

Al observar la aplicación de la metodología, no todos los docentes lo aplican de manera homogénea, se observa que algunos de ellos tienden a aplicar aspectos del sistema de educación tradicional. Un aspecto positivo del conocimiento del modelo, es la claridad que tienen los docentes por el respeto de los ritmos de aprendizaje de los estudiantes y la aplicación de los principios de la pedagogía activa. Pero argumentan la falta de tiempo para atender las particularidades de los ritmo de aprendizaje de una serie de estudiantes, que se deben muchas veces a problemáticas concretas de ellos, como la inasistencia. Los docentes afirman que el modelo exige del estudiante mucha disciplina tanto en asistencia, como disponibilidad, entrega, entusiasmo, interés, etc.

La aplicación del modelo escuela nueva requiere de una serie de estrategias metodológicas, que muchas veces el docente carece, que va desde la organización del salón de clase, en donde se ordenan los pupitres de la forma tradicional, dirigidos hacia un docentes que hace uso de las conferencias, pero la organización del salón de la clase debe de ser más democrático en forma 
de semicírculo donde todos los participantes puedan verse frente a frente.

Es general la opinión de los docentes facilitadores que necesitan más capacitación en el modelo pedagógico de Escuela Nueva, principalmente en el desarrollo de las competencias y en las formas evaluativas, sobre aspectos como la autoevaluación, la coevaluación, la heteroevaluación.

Pero el avance se va logrando cuando el docente invita a los estudiantes al análisis, a la reflexión, al cuestionamiento sobre la realidad y la construcción del conocimiento. Seguir las guías modulares, permite iniciar con la vivencia que tiene el estudiante, que luego profundiza y busca respuesta en la fundamentación científica. Las lecturas que encuentra en la fundamentación científica deben de generar discusión, interpretación y análisis, el docente pone en práctica la creatividad para que sus estudiantes hagan uso de su propio conocimiento, de otras fuentes de conocimiento, del diccionario, del debate, de los plenarios, de la explicación, de la utilización de estrategias creativas como los sociodramas, entre otros.

\section{Saber ser}

Una categoría importante en el modelo Escuela Nueva, es el saber ser, que son las características actitudinales personales que posee el docente y que mediante el ejemplo trasmite a los educandos, aspectos personales como tener madurez, responsabilidad, principios, valores, compromiso, amor, seguridad, autoestima, empatía, imaginación, entusiasmo, optimismo, liderazgo, emprendedurismo, solidaridad, compañerismo, innovación, creencias, entre otros. Son una serie de aptitudes que permitirán el crecimiento humano, contribuyendo a la formación holística e integral de los estudiantes.

Aspectos como ser responsable con el tiempo programado para las clases de parte del docente son características positivas. El $50 \%$ de los estudiantes participantes en el grupo focal, determinan que los docentes no cumplen con el tiempo asignado y generalmente pierden de 10 a 30 minutos, al terminar sus clases antes del límite fijado. Aunque el $100 \%$ de los docentes entrevistados indican que cumplen con el tiempo asignado para el desarrollo de sus clases.

Otros valores que según los docentes cultivan en los estudiantes, son: la entrega y vocación de servicio a la humanidad, el trabajo en equipo, el trabajo colaborativo, la responsabilidad en el cumplimiento de sus tareas extraescolares, edificaran al estudiante en su compromiso y responsabilidad. Pero si estas características no se observan en los docentes facilitadores, difícilmente se formaran estudiantes integrales, porque no ven el ejemplo en los facilitadores.

Los estudiantes definen que la ética es una característica que frecuentemente se deben de observar en los docentes, como la tolerancia, la humildad, el respeto a la diversidad cultural, la objetividad, el respeto a la madre tierra, la imparcialidad, la neutralidad, la autonomía, el derecho ajeno, el respeto a la sabiduría popular, el fomento de la cordialidad entre estudiantes, entre estudiante y docente, entre docentes y familias rurales, etc. Los docentes facilitadores reconocen que en el proceso de aprendizaje con los estudiantes, ellos no son los dueños de conocimiento, debido a que en el intercambio también aprenden de sus estudiantes.

Los docentes consideran que es importante en el saber ser, donde se debe fomentar la formación del pensamiento crítico autónomo, requiere del desarrollo de valores como fomentar el diálogo con el otro, ya sea compañero o docente y en este proceso, a medida que los estudiantes van madurando su pensamiento, adquieren la capacidad para colocarse en el lugar del otro y de esta manera, entender sus razones. Lo anterior explica muy bien la importancia de un ambiente de aprendizaje donde se permita la comunicación entre los distintos actores que se encuentran en el aula de clase. En este sentido se percibe en el grupo focal que todavía los estudiantes no poseen la total apropiación que ellos en 
este modelo educativo tienen el protagonismo debido a que son el centro de proceso de enseñanza aprendizaje.

\section{Saber hacer}

Esta categoría saber hacer, es la base de la escuela activa, cuyo sinónimo es escuela nueva. El saber hacer es la formación procedimental, que guía la aplicación práctica de los conocimientos. Tampoco debe tomarse como sinónimo de competencia, debido a que algunos consideran que el desarrollo de competencia es la realización mecánica de algunas actividades, pero la formación basada en competencia atiende el saber hacer, pero también el saber conocer, el saber ser y el saber a convivir, siendo las competencia una formación integral del discente.

Para los docentes, el principio de la escuela activa, es cuando el estudiante no se limita a ser un simple receptor de los conocimientos, sino que se vuelve activo, participativo, propone un proyecto, presenta su idea, un prototipo, que innova, en vez de limitarse a ser un espectador o acumulador del conocimiento.

Cuando se ejecutan las clases de la carrera de Desarrollo Rural Sostenible, siguiendo el desarrollo de las guías modulares, en los momentos pedagógicos de ejercitación y aplicación, de cada una de las unidades, se debe efectuar la actividad práctica, la primera en el aula y la segunda en las comunidades, en las fincas. Por lo tanto el saber hacer se realiza al implementar el modelo de Escuela Nueva.

La ejercitación es la parte practica de la teoría, es su interpretación, es flexible en su aplicación y se desarrolla en las aulas de clase, pero en múltiples ocasiones los estudiantes se encuentran realizándolas junto a su equipo de trabajo en los corredores de los pabellones, debajo de los árboles, en las áreas verdes, en las áreas deportivas, en otras aulas que se encuentran desocupadas, etc. Lo importante es la comodidad de los estudiantes para la realización de este acápite, pero con el cumplimiento de tiempo determinado para la actividad. Tanto docentes como estudiantes acreditan una gran importancia en el desarrollo de esta actividad. Hay un señalamiento de los estudiantes donde afirman que existen docentes que no realizan esta actividad o que no le da la debida importancia.

Los docentes indican, que según la unidad y modulo que se este desarrollando, que las actividades de la aplicación, muchas de ellas son orientadas de forma individual o colectiva, y que se realizan en las unidades productivas, en las comunidades y en otras ocasiones están dirigidas a realizarse con el docente y con toda la clase, en el caso de visitas o giras técnicas.

Los estudiantes afirman que en la aplicación, según el caso se requieren de resolver problemas, de hacer investigaciones con sus familias o en sus comunidades, en resolver estudios de casos, en visitar unidades productivas o instituciones en su entorno municipal, en buscar a actores comunales que les brinde una información concreta, o bien la realización de actividades prácticas en áreas como huertos individuales o colectivos, en visita para conocer otras experiencias productivas, organizativas o de innovación socioproductivas.

Los estudiantes expresaron que en pocas asignaturas, aunque las guías modulares presentan muchas actividades prácticas, que son acciones dinámicas, interesantes e importantes en el desarrollo del aprendizaje, estas actividades se desarrollan en el aula de clase y se vuelven rutinarias, aburridas, con aplicación de educación tradicional. Aunque no es la mayoría de los docentes que lo realizan, pero existe la inquietud de los estudiantes, que además reclaman más actividad práctica en la formación como profesionales en desarrollo rural.

La evaluación del aprendizaje por parte de los docentes facilitadores está basada en como los estudiantes realizan las acciones de ejercitación y aplicación, propuesta en las guías modulares. Muchos los cumplen como está orientado, pero existen algunos que orientan 
las actividades de ejercitación y aplicación, pero en la siguiente sección de clase, no es retomada a través de un plenario, a como esta descrito en la guía modular. Todavía hay docentes según sus propias afirmaciones que no realizan evaluación cuantitativa valorando las actividades del saber hacer, y priorizan la evaluación del conocimiento científico teórico, del saber conocer.

Estudiantes y docentes, consideran que el saber hacer es una parte fundamental de la formación y es una experiencia muy interesante, porque recupera la práctica diaria, la relaciona con el entorno y se hace un ejercicio efectivo en las condiciones reales de las comunidades, no es un espacio utópico definidos en los contenidos sino que se va a poner en marcha el saber hacer para el logro de las capacidades según con la realidad que se encontrará en el desarrollo en su campo profesional, permitiéndole integrarse de una manera efectiva.

\section{Saber convivir}

El saber convivir, es consolidar en los docentes y estudiantes de valores como la tolerancia, en el convivir, cohabitar con alguien que no comparte nuestras ideas, que es parte de la diversidad tanto de ideas, de comportamientos, de acciones, pero se debe de convivir sin llegar a la confrontación, se trata de buscar e identificar los puntos comunes y dialogar en el campo del respeto.

Los estudiantes explican que los docentes respetan sus ideas políticas, religiosas, culturales, puntos de vista divergentes como el género, la diversidad cultural, la diversidad sexual, facilitan e impulsan acciones que unen el grupo a través de consenso.

Los docentes facilitadores admiten que implementan el saber convivir, pero que es difícil su implementación, porque existen educandos que tienen un punto de vista definido, y no ceden en sus posiciones, pero que poco a poco han iniciado un proceso de tolerancia, impulsado por el dinamismo del grupo, que conduce a aceptar la diversidad. La convivencia se evidencia en las elecciones de los gobiernos estudiantiles, en el freno a las acciones de maltrato estudiantil (bulín). Este saber debe practicarse en acciones como saber participar, dando igual oportunidad a todos, en saber cooperar, en saber ser solidario, en evitar el protagonismo, en saber ser responsable consigo mismo, con su formación y con sus compañeros. Los facilitadores terminan concluyendo que todo se resume en ser tolerantes como base del saber convivir.

Por su lado los estudiantes hacen referencia que en la convivencia, hay docentes que se aferran a posiciones apegadas a los descrito en los contenidos científicos y que no le dan valor a los conocimientos empíricos y ancestrales. Muchos no toman en cuenta las condiciones sociales, económicas, productivas, ambientales, del territorio de incidencia. Señalan que en algunos docentes se evidencia la intolerancia, al no comprender las condiciones de los estudiantes, en cuanto al no disponer de Internet, muchas veces al no disponer de energía eléctrica, a lo alejado de sus comunidades, a las llegadas tardes por problemas en el transporte y en el camino. No comprenden a los estudiantes cuando se presentan enfermedades o algún familiar está enfermo. Incluso afirman que hay docentes que han llegado a expresar a los estudiantes, para que si tenían ese problema se han decidido estudiar.

Expresiones de estudiantes hacia los docentes, sobre la falta de convivencia, están: "quieren que le repita en las pruebas, lo que esta anotado en el modulo", "cuando le pido que repita un contenido que no entendí, me dice que eso ya paso, que no puse atención, que eso lo debería de saber", "el profesor no me comprende que tengo serias limitante con el uso de la tecnología", "permite que mis compañeros de clase, no me dejen hablar y cuando lo hago, me hacen burla".

\section{Saber convivir con el medio ambiente}

Los estudiantes afirman tener una buena base en los aspectos ambientales, evidenciados en los módulos 
recibidos como: Ecología, Manejo y Conservación de los Recursos Naturales, Biodiversidad, Sistemas Agroforestales, entre otros, pero los docentes afirman que en la mayoría de los módulos hay un eje transversal muy bien definido que insiste en la producción en armonía con el medio ambiente, que ha desarrollado un buen nivel de conciencia hacia el cuido del mismo.

La convivencia con el medio ambiente, se basa en demostrar los estragos que tenemos en todos los factores del ambiente, donde se ven reducido los niveles de precipitaciones, se ha disminuido los niveles hidrológicos con fluentes hídricas en proceso de degradación, se ha incrementado la desforestación, se ha aumentado la temperatura ambiental y todo esto impacta en la producción primaria. Ellos están conscientes de esta problemática y se están integrando a campañas a favor del medio ambiente, principalmente a reforestaciones y en especial a la defensa del bosque y las reservas que posee el municipio.

Los estudiantes definen que la conciencia ambientalista que poseen se evidencia en la unidad de producción con un uso racional de los agroquímicos, en mantener el uso de semillas criollas, en la protección de los suelos y aguas, en evitar el avance de la frontera agrícola, en el uso de tecnologías apropiadas, en la protección arbórea de las fuentes hídricas, en evitar la contaminación de las fuentes hídricas con aguas mieles y con pulpa de café, en el uso de subproductos como residuos de cosechas, estiércoles, convirtiéndolos en abonos orgánicos. Los docentes indican que uno de los ejes disciplinares de la carrera es el ambiental, indicando que desde ahí se fortalece el saber convivir con el medio ambiente, máximo en territorios que son vitales para la nación en mantener las áreas protegidas que poseen, y mantener los sistemas productivos agroforestales como es el cultivo del café.

\section{CONCLUSIONES}

Para el desarrollo de competencias en los estudiantes, los docentes facilitadores deben de reunir una serie de competencias, en el presente estudio se encontró:

El saber conocer, los docentes poseen una buena preparación en conocimientos teóricos científicos, integran proceso de la investigación científica aunque con limitantes, orientan la implementación de las innovaciones tecnológicas, pero es necesario mejorar la metodología de enseñanza aprendizaje y de evaluación, dentro del modelo Escuela Nueva.

En el saber ser, se desarrollan valores y principios éticos, como parte de la formación integral del estudiante, aunque se debe de mejorar algunos detalles.

En el saber hacer, parte importante del aprendizaje procedimental y de la aplicación práctica de los conocimientos, que permite desarrollar competencias profesionalizante, debe tenerse el cuidado de que no se vuelva una rutina mecánica de las actividades. Deben de realizarse todas las actividades orientadas en las guías modulares en los aspectos de ejercitación y aplicación.

Saber convivir, es desarrollar la competencia de la tolerancia, como medio de lograr el desarrollo a través del consenso, es necesario fortalecer según los estudiantes los aspectos de convivencia, comprensión y tolerancia.

Saber convivir con el medio ambiente, es reconocer que toda actividad del hombre tiene un impacto en la naturaleza, pero se debe de incidir en que el impacto sea lo menos posible, sin causar daños mayores a la madre tierra, de forma que se trabaje en armonía con el medio ambiente.

\section{RECOMENDACIONES}

La principal recomendación, es que se debe fortalecer los conocimientos de las metodologías activas y del Modelo Escuela Nueva a los docentes facilitadores, a través de capacitaciones sistemáticas, así como 
también sobre la conceptualización teórica y práctica de las competencias.

\section{AGRADECIMIENTOS}

Se agradece a los estudiantes activos del programa Universidad en el Campo (UNICAM) de la carrera de Desarrollo Rural Sostenible por el apoyo en la recolección de la información y a los docentes facilitadores que cordialmente nos brindaron sus entrevistas.

\section{REFERENCIAS BIBLIOGRÁFICAS}

Arenas C. (2016). Habilidades del pensamiento: Una posibilidad para la enseñanza aprendizaje de nomenclatura inorgánica. Maestría en Enseñanza de las Ciencias Exactas y Naturales. Colombia: Universidad Nacional de Colombia, Sede Manizales.

Bravo, N. (2007). Competencias Tuning Europa, Tuning America Latina. Basado en informes reuniones del Proyecto Tuning Europa America Latina. Colombia: Bogotá. Disponible en: http:// www.cca.org.mx/profesores/cursos/hmfbcp_ut/ $\mathrm{pdfs} / \mathrm{m} 1 /$ competencias_proyectotuning.pdf Consultado el 14-02-2016

Colbert, V. (1999). Mejorando el acceso y la calidad de la educación para el sector rural pobre. El caso de la Escuela Nueva en Colombia. Organización de Estados Iberoamericanos. Revista Iberoamericana de Educación. No 20. Pág. 107-135.

Conde, SD. (2008). Las competencias sociales y los indicadores en Ingenierías. UNAN Managua.

Dirección General de Educación Superior para Profesionales de la Educación (DGESPE) (2009). Modelo curricular para la formación profesional de los docentes de educación básica. Mexico: Secretaria de Educación Pública. Subsecretaria de Educación Superior.

Duarte, J. (2010). Ambientes de Aprendizajes. Una aproximación conceptual. Colombia: Universidad de Antioquia. Revista Iberoamericana de Educación (ISSN: 1681-5653)

Hidalgo, J. \& Marín, M. (2003). Competencias: Saber hacer, ¿en cuál contexto? Capítulo del libro: El concepto de competencia II. Una mirada interdisciplinar. Colombia, Bogotá: Sociedad Colombia de Pedagogía.

Johnson, D. (1999). El aprendizaje Cooperativo en el Aula. Buenos Aires, Argentina: Ed. Paidós Educador.

Lacki, P. (SF). Los agricultores necesitan de un sistema educativo que les ayude a solucionar sus problemas. Disponible en línea: http://www.polanlacki.com.br Consultado el 03-01-2015

Mejía, M. (2006). Educación(es) en la(s) globalización(es) I. Colombia, Bogotá: Ediciones Desde Abajo.

MEN/ASCOFADE-Ministerio de Educación Nacional/Asociación Colombiana de Facultades de Educación (2005). Talleres de apropiación de los estándares de competencias básicas y ciudadanas. Colombia, Bogotá: Enlace. Proyecto MEN/ ASCOFADE.

Mialaret, G. (1977). Ciencias de la Educación. España: Barcelona. Edit. Oikos-tau, S.A.

Mogollón, O. \& Solano, M. (2011). Escuelas Activas. Apuestas para Mejorar la Calidad de la Educación. FHI360. Washington, DC., USA.

Pérez, G. (2011). Estructura del desempeño idóneo: saber hacer, saber conocer y saber ser en la formación por competencia. Venezuela: U.E. Santiago F. Machado.

Ramón, JN. (2010). Formación de competencias para salir de la pobreza en modelos educativos rurales. Argentina: Primera Edición, Buenos Aires: Consejo Latinoamericano de Ciencias Sociales - CLACSO. Universidad de Caldas (2011). Documento Maestro del Técnico Profesional en Desarrollo Rural Sostenible del Proyecto ALFA III, Universidad en el Campo. Colombia. 